\title{
Qa-2 expression levels is related with tumor-infiltrating lymphocytes profile during solid Ehrlich tumor development
}

Qa-2 and TILs relationship in breast cancer model

Istéfani Luciene Dayse da Silva ${ }^{1}$, Emerson Soares Veloso ${ }^{1}$ Ivy Nayra Gonçalves Nascimento $^{1}$, Ariadne Duarte Braga ${ }^{2}$, Miriam Teresa Paz Lopes ${ }^{2}$, Miguel Quintnilla ${ }^{3}$, Renata Toscano Simões ${ }^{4}$, Enio Ferreira ${ }^{1 *}$.

1 - Department of General Pathology, Laboratory of Comparative Pathology, Biological Sciences Institute, Federal University of Minas Gerais, 486, 31270-901 Belo Horizonte, Minas Gerais, Brazil

2 - Department of Pharmacology, Laboratory of Antitumor Substances, Biological Sciences Institute, Federal University of Minas Gerais, 486, 31270-901, Belo Horizonte, Minas Gerais, Brazil

3 - Departament of Cancer Biology, Instituto de Investigaciones Biomédicas “Alberto Sols” - Consejo Superior de Investigaciones Científicas - Universidad Autónoma de Madrid, 4, 28029, Madrid, Spain

4 - Institute of Education and Research of Santa Casa of Belo Horizonte, 590, 30150240, Belo Horizonte, Minas Gerais, Brazil

* Corresponding author: Enio Ferreira. Department of General Pathology, Laboratory of Comparative Pathology, Biological Sciences Institute, Federal University of Minas Gerais, 486, 31270-901, Belo Horizonte, Minas Gerais, Brazil. Tel.: +5531 3409 3039; Fax: +5531 3409 2873. e-mail address: enioferreira@icb.ufmg.br 


\section{Summary}

Breast cancer is an immunogenic tumor and presence of tumor-infiltrating lymphocytes (TILs) has been proposed as prognostic and predictive biomarkers. Likewise, Human Leucocyte Antigen G (HLA-G) has been proposed as bad biomarker in several cancer types. However, absence of a model to study HLA-G, limit the full understanding of the mechanisms involved. In this way, the aim of this study was to describe the pattern of Qa-2 expression and its relationship with the profile of tumor-infiltrating lymphocytes in solid Ehrlich tumor. The Ehrlich tumor growth was evaluated in Balb/c female mice in different tumor stages. The inflammatory infiltration features was determined by histopathology and, lymphocyte type, tissue Qa-2 expression and CDC47, by immunohistochemistry. ELISA kit was used to determine Qa-2 expression in the serum from the animals. We observed that, Qa-2 in neoplastic cells increases in initial tumor development stages, while, serum Qa-2 increase in the late stage. Qa-2 increasing is correlated with CD3+ increase and proliferation index decrease in neoplastic cells. Our results suggest that Qa-2 expression is related to the Ehrlich tumor development by immune response modulation.

Keywords: solid Ehrlich tumor, Histopathology, Immunohistochemistry, breast cancer, tumor immunology and mice. 


\section{Introduction}

Breast cancer is the most common cancer among women in developing country (Delort, et al., 2015, Engels, et al., 2015). This is responsible for many deaths in worldwide (Romo-Gonzalez, et al., 2015). Treatment consists of surgery, radiotherapy, and chemotherapy. However, patient particularities can make treatment difficult. Many studies show that host's cellular immune response plays a pivotal role in controlling tumor development (Agaugue, et al., 2011, Matkowski, et al., 2009, Sato, et al., 2005).

Neoplastic transformation causes changes in tissue architecture and promotes the activation of the immune response to eliminate the tumor. However, transformed cells escape from this response. This process is known as cancer immunoediting and it has been related with prevention and promotion of several cancers types (Dushyanthen, et al., 2015, Mittal, et al., 2014).

Breast cancer is an immunogenic tumor and presence of tumor-infiltrating lymphocytes (TILs) in this, has been proposed as prognostic and predictive biomarkers (Wesolowski and Carson, 2014). Studies describe that high number of TILs is associated to better survival and therapy response (Cimino-Mathews, et al., 2015, Mao, et al., 2014, Tung and Winer, 2015). On the other hand, the expression of immune modulating molecules like Human Leucocyte Antigen G (HLA-G) is associated with immune evasion and poor prognosis (Curigliano, et al., 2013). This HLA-G mediated immune evasion has been associated with immune cell inhibition directly through inhibitory receptors engagement, and indirectly through suppressor/regulatory cells induction or by trogocitosis (Carosella, et al., 2015). However, a few quantity models are available to in vivo studies. Many models limit the full understanding of the mechanisms involved, like xenografts models, and difficult the realization of functionally studies (Richmond and Su, 2008). 
Qa-2 has been proposed as a murine HLA-G homologue in gene structure, protein conformation and functionality (Cao, et al., 1999, Comiskey, et al., 2003, Lee, et al., 2010), though its function during tumor development remains unclear (Chiang, et al., 2002, Chiang and Stroynowski, 2004).

Solid Ehrlich tumor is one of the commonest transplantable breast cancer model. Advantages of this cancer model are fast growth in an immune-competent host, low cost, and easy monitoring (Kabel, et al., 2015). These characteristics make solid Ehrlich carcinoma a good model to study the involvement of Qa-2 in breast cancer immunosurveillance. Thus, the aim of this study was to describe the pattern of Qa-2 expression and its relationship with the profile of tumor-infiltrating $\mathrm{T}$ lymphocytes during Ehrlich tumor development. This work is the first to describe the expression of Qa-2 in a syngeneic breast cancer model and provides information to clear up the Qa-2 role in cancer immunosurveillance.

\section{Material and methods}

Animals and groups

Female Balb/c mice (6 weeks, 20 g weights) were provided by the Institute of Biological Science of the Federal University of Minas Gerais, Belo Horizonte, Minas Gerais, Brazil. The animals were housed in standard conditions $\left(24^{\circ} \mathrm{C}\right.$ temperature and 12h light/12h dark cycles) and allowed to acclimatize for 1 week before starting the experiments. The ethical committees of local Institutional Animal Welfare Committee approved the study, under protocol number 350/2012.

The animals were divided into two groups: Ehrlich (EHR; n=26) and Control (CTRL; $n=26$ ). Animals were euthanized on three different days after inoculation: 2 
(EHR-2 days and CTRL-2 days), 7 (EHR-7 days and CTRL-7 days) and 14 (EHR-14 days and CTRL-14 days).

Cells

The Ehrlich tumor was grown as ascites in mice. For the experimental procedures, $3 \mathrm{~mL}$ of ascitic fluid from mice, inoculated 12 days before, was collected and centrifuged ( 3 min, $200 \mathrm{x}$ g). The supernatant was removed and Ehrlich tumor cells were washed three times in saline. Viability was determined by the Trypan blue dye exclusion test and counted in a modified Neubauer chamber ( $>90 \%$ ) to post inoculation.

\section{Experimental protocol}

Animals of the Ehrlich group were inoculated with $2.5 \times 10^{6}$ Ehrlich tumor cells (in a final volume of $50 \mu \mathrm{L}$ ) in the left footpad and Control group was inoculated with saline $(50 \mu \mathrm{L})$ in the same field and underwent the same procedures.

Weights of animals and tumor sizes were determined periodically. To assess the Ehrlich tumor growth curve, measurements were performed using a micrometer (Mitutoyo, measurement $0.01 \mathrm{~mm}$, series n. 7301) immediately before and every two days after tumor inoculation, with a total of eight measurements (Ferreira, et al., 2007).

After the animals had been euthanized, necropsy was performed by removing the tumor, lymph nodes, heart, liver, brain, spleen, and lungs from all animals for histopathological and immunohistochemistry analysis to Qa-2. Blood samples from all animals were also collected for quantification of seric Qa-2 expression.

\section{Histopathology}


Histologic sections of the tumor, lymph-nodes, heart, liver, brain, spleen, and lungs stained with hematoxylin and eosin (HE) were used to evaluate metastases and classify peritumoral inflammatory infiltrate. Tissues were fixed in formalin $(10 \% \mathrm{w} / \mathrm{v}$ in phosphate-buffered saline - PBS pH 7.4). Peritumoral inflammatory evaluation was performed considering three factors: distribution (focal or diffuse), intensity (light, moderate or heavy), and profile (mononuclear, polymorphonuclear or mixed mononuclear and polymorphonuclear). Even, intratumorally inflammatory infiltrate was evaluated to intensity (light, moderate or heavy).

\section{Immunohistochemistry}

A peroxidase system was used in the immunohistochemistry procedure was used to identify the secondary antibody by polymer (ADVANCE HRP). Antigen retrieval was performed in water bath at $98^{\circ} \mathrm{C}$, with a citrate buffer solution $(\mathrm{pH} \mathrm{6.0)}$ (Target Retrieval Solution) for 20 minutes. To block the endogenous peroxidase activity, slides were incubated with a solution of $\mathrm{H}_{2} \mathrm{O}_{2} 3 \%$ in methyl alcohol. Reagents were applied manually, consisting of 1 hour incubation for the primary antibody and 30 minutes for the reagents, except for the chromogen diaminobenzidine, incubated for 1 minute. Positive and negative controls were included in each bath. The antibodies used were: Anti-Qa-2 (clone 69H1-9-9; eBioscience, dilution 1:100); Anti-CD4 (clone GK1.5; eBioscience, dilution 1:100), Anti-CD8 (clone 4SM15, eBioscience, dilution 1:100), Anti-CD3 (clone CD3-12; UC-Davis, dilution: 1:500), Anti-Foxp3+ (clone sc-53876, Santa Cruz Biotechnologies, dilution 1:600), Anti-CDC47 (clone 47DC141; Neomarkers, dilution 1:300).

The expression of Qa-2 was analyzed separately in inflammatory cells and neoplastic cells. The index for Qa-2 staining in inflammatory peritumoral cells was 
obtained by mean of positive cells in 15 fields (60x). Tumor cell Qa-2 staining was classified in: +1 when up to $10 \%$ of cells staining; +2 when $11-25 \%$ of cells staining, and +3 when more than $25 \%$ of cells staining. The CDC47 index was obtained by percentage of positive cells in 1000 tumor cells. The CD4, CD8, CD3 and Foxp3+ index was obtained by mean of positive cells in 10 fields (40x).

\section{ELISA}

To evaluate the seric Qa-2 expression after collection, the samples were centrifuged for 5 minutes at $3000 \mathrm{rpm}$ and the serum aliquoted and stored in a plastic Eppendorf tube.

A specific ELISA kit was used for the Qa-2 determination (Qa lymphocyte antigen 2 region, Qa-2, Qa-2 Elisa Kit - MyBiosource.com). All the manufacturer's recommendations were followed. Samples were done in duplicate and the standard curve values were determined from concentration to Qa-2.

\section{Statistical Analysis}

Results are presented as mean \pm standard error of mean (SEM). The data were tested for normality using the Kolmogorov-Smirnov test. The outliers were identified using the Grubbs' test by outlier calculator on GraphPad QuickCalcs online. Comparisons were carried out using Student's t-test and ANOVA with Bartllet's test and Bonferroni’s Multiple Comparison Test for comparing groups, test Kruskal-Wallis with Dunn's test to unpaired data, Cochran Q test to compare groups with dichotomous variables, and Spearman's correlation. A P-value less than 0.05 was considered significant. The statistical programs used to analyze the data were GraphPad InStat version 5.0 and BioStat Professional version 5.8.4. 


\section{Results}

\section{Tumor behavior and histopathology evaluation}

Upon transplantation of Ehrlich carcinoma cells into the footpad of Balb/c mice, a significant progressive increase of tumor size and weight gain was observed (Fig. 1AC). Statistical differences in the size of tumors were observed between different times post-inoculation, indicating a rapid growth of tumors (Fig. 1B). This resulted in a significant difference in the weight of the animals between 2 and 14 days postinoculation (Fig. 2C). Complete data is available in supporting information Table 1s.

Tumors were located deep into the dermis invading the musculature, and sheets of small, higher chromatophilic tumor cells of variable shapes representing cell proliferation were observed surrounding areas of necrosis and differentiated cells (data not shown).

Nuclear CDC47 staining was observed in neoplastic cells at all times postinoculation (Fig. 2A). Proliferation index values obtained from CDC47 staining showed a maximum at 2 days and a significant reduction at 7 and 14 days post-inoculation $(\mathrm{P}=0.02)$ (Fig. 2B and Table 2s).

The peritumor inflammatory infiltrate was diffuse at all post-inoculation times, mainly composed of polymorphonuclear and mononuclear leukocytes. The intensity of peritumor inflammatory infiltrate was predominantly moderate, with significant difference between 2 and 7 days post-inoculation $(\mathrm{P}=0.01)$. Intense inflammation was not observed (Fig. 3A and B). A positive correlation was observed between the animal weight and the intensity of peritumoral inflammatory infiltrate ( $\mathrm{r}=0.4032 ; \mathrm{P}=0.04)$. 
The intratumorally infiltrate was rather weak with a significant difference between 2 and 7 days post-inoculation $(\mathrm{P}=0.02)$ (Fig. 3C; complete data is available on supporting Table 3s). A positive correlation was observed between tumor size and intensity of intratumorally inflammatory infiltrate $(\mathrm{r}=0.4340, \mathrm{P}=0.03)$. Metastasis were observed only in regional lymph nodes at 14 days post-inoculation $(n=4 / 9 ; 44 \%$ - data not shown).

\section{Lymphocyte infiltrate profile}

Immunohistochemistry to discriminate the types of lymphocytes infiltrating the tumors showed cytoplasmic staining to $\mathrm{CD} 3+, \mathrm{CD} 4+, \mathrm{CD} 8+$ and Foxp3+ surface markers (Fig. 4A-D). Quantification showed progressive increase of CD3+ lymphocytes with tumor development. In contrast, CD4+ lymphocytes decreased at a similar rate. Differences were statically significant for CD3+ lymphocytes between 2 and 14 days post-inoculation $(\mathrm{P}=0.02)$, and for $\mathrm{CD} 4+$ lymphocytes between 2 and 14 days postinoculation ( $\mathrm{P}=0.04)$ (Fig. 5A and $\mathrm{B}$ ). The presence of $\mathrm{CD} 3+$ lymphocyte was positively correlated with tumor size $(r=0.4601$; $P=0.03)$, while the profile of $C D 4+$ lymphocytes showed a negative correlation $(\mathrm{r}=-0.5967 ; \mathrm{P}=0.003)$. An inverse profile was observed between CD8+ and Foxp3+ lymphocytes, with CD8+ lymphocytes decreasing and Foxp3+ lymphocytes increasing at day 7 (Fig. 5C, D and Table 4s).

\section{Qa-2 levels in neoplastic cells, peritumor inflammatory cells and serum}

Qa-2 expression in neoplastic cells was always focal, often seen at the invasive front, and accounting for no more than 25\% of tumor cells (Fig. 6A). Qa-2 expression increased on day 7 with respect to day 2 post-inoculation and was reduced at day 14 
(Fig. 6B), with significant difference when comparing 2 and 7 days post-inoculation ( $\mathrm{P}=0.006$ ) (See supporting Table 5s). Positive correlation was observed between Qa-2 expression in neoplastic cells and the presence of CD3+ lymphocytes $(r=0.5282$; $\mathrm{P}=0.02$ ). In contrast, CDC47 expression was negatively correlated with Qa-2 expression in neoplastic cells ( $\mathrm{r}=-0.6029 ; \mathrm{P}=0.02)$.

Qa-2 expression in inflammatory cells from peritumor inflammatory infiltrate increased on day 7 with respect to days 2 and 14 post-inoculation; however, these differences were not statistically significant (Fig. 6C). The serum levels of soluble Qa-2 increased progressively during tumor growth. The enhancement observed in the sera of animals bearing tumors at day 14 post-inoculation with respect to control (tumor-free) animals was statistically ( $\mathrm{P}=0.003$ ) (Fig. $6 \mathrm{D}$ and supporting Table 6s). Other correlations were not observed.

\section{Discussion}

Innate and adaptive immune responses protect the host against the development of cancer. Furthermore, tumor cells can evade immunosurveillance through many mechanism, such as HLA-G expression (Loumagne, et al., 2014). Qa-2 has been proposed as HLA-G homolog in embryos implantation (Cao, Brenner, Alikani, Cohen and Warner, 1999, Comiskey, Goldstein, De Fazio, Mammolenti, Newmark and Warner, 2003) and autoimmune disease (Lee, Choi, Kwon, Shim, Park, Lee and Sohn, 2010). However their role in cancer models remains unclear. Sometimes it is described as a functional homolog (Chiang, Henson and Stroynowski, 2002), and other times as opposite of it (Chiang and Stroynowski, 2004). 
We described for the first time the profile of Qa-2 expression in a breast cancer model. In addition, we characterized the Qa-2 relationship with tumor lymphocytes infiltrate profile. Our results demonstrate a moderate inflammatory intensity in initial tumor development stage, both intratumorally and peritumor. Interestingly, this correlates with an increase of Qa-2 expression in neoplastic cells, suggesting that Qa-2 may be expressed in order to modulate the immune response intensity at this development time. Matching to what we could observe in this work, the direct relationship between breast cancer luminal type and TILs increasing has been already described previously in a work with humans and HLA-G (Dong, et al., 2012).

On the other hand, the increase of seric Qa-2 levels in late tumor development stage is associated with size tumor increase and inflammatory intensity reduction, suggesting that, the soluble and membrane Qa-2 behavior can be different in immune modulation role, than transmembrane Qa-2. These findings corroborate with what is already described for HLA-G, which soluble isoform have an important role in immune cells chemotaxis (Morandi, et al., 2014). Furthermore, soluble Qa-2 may have a late expression, or detectable increase levels in the late tumor development stage. It is possible by transmembrane Qa-2 loss or by proteolytic shedding (Park, et al., 2004), where the transmembrane isoform can be cut by metalloproteinases and generate soluble isoform detectable in the serum (Rizzo, et al., 2013). Therefore, we hypothesize that soluble and transmembrane Qa-2 isoforms have complementary role in tumor immune evasion. In initial tumor development stages, the mainly isoform related to immune evasion and tumor progression is the transmembrane Qa-2, while in late tumor development stages this role is granted to the soluble Qa-2. These two isoforms together may inhibit immune cells and alter the profile of infiltrated inflammatory cells. 
Inflammation play double role in tumor development and may be considered as a good or bad prognosis (Dushyanthen, Beavis, Savas, Teo, Zhou, Mansour, Darcy and Loi, 2015, Marshall, et al., 2016, Mittal, Gubin, Schreiber and Smyth, 2014). Corroborating, our results suggest that CD3+ and CD4+ inflammatory infiltrate play an opposite role in Ehrlich tumor development, where CD3+ was correlated with tumor size increase and CD4+ with tumor size decrease. Furthermore, Qa-2 expression in neoplastic cells was positively correlated with CD3+ increasing. Although not significant, all these findings occur in parallel to the enhancement of Foxp3+ lymphocytes, and the decrease of CD8+ lymphocytes, which may indicate an increase of $\mathrm{T}$ regulatory cells induction. Probably, CD3+ increase can be related to regulatory cells induction by Qa-2 (Pankratz, et al., 2014), since, just Foxp3+ cells don’t represent the entire panel of regulatory cells (Djurisic, et al., 2015, Li, et al., 2013).

We believe that, Qa-2 can reduce CD4+ and CD8+ cells chemotaxis by inhibitor receptors ligation (Naji, et al., 2007, Naji, et al., 2007). Besides that, Qa-2 expression can be related to the cytokine balance in favor of Th2 immune response, facilitating tumor immune evasion, same as described to HLA-G (Agaugue, Carosella and RouasFreiss, 2011).

Still, we demonstrate negative correlation between CDC47 expression and Qa-2 expression on neoplastic cells. Likely, Qa-2 can regulate proliferation status by specific pathways, such as, src quinases activation (Comiskey, et al., 2007, De Fazio and Warner, 2007), STAT3 activation (Liu, et al., 2013), or indirectly by induction of IL-6, IL-8 and TNF $\alpha$ cytokines (Jiang, et al., 2015, Li, et al., 2009). However, proliferation index is not always related to aggressiveness. Several other features are responsible to aggressiveness status, such as invasiveness and migration (Yang, et al., 2016). Dormancy status has been associated with stemness features and poor prognosis (Kreso 
and Dick, 2014, Smalley, et al., 2013). Therefore, to explore the exact impact of Qa-2 in neoplastic cell proliferation and stemness features, an in vivo test for the function evaluation must be carried out.

In conclusion, our results suggest that transmembrane and soluble Qa-2 have complementary role in the solid Ehrlich tumor development by induction of CD3+ and Foxp3+ increase and CD4+ and CD8+ decrease. Moreover, solid Ehrlich tumor model can assist in understanding the role of HLA-G function in breast cancer development. 


\section{Conflict of Interest}

The authors declare that there is no conflict of interests regarding the publication of this paper.

\section{Acknowledgments}

This paper was supported by a grant from CAPES Foundation (Higher Level Personnel Improvement Commission), with an International Scholarship Procs. Nr. 99999.009972/2014-05. Other grants were also provided by CNPq (Brazilian National Research and Development Council) and FAPEMIG (Foundation for Research Support of the State of Minas Gerais). 


\section{Reference}

Agaugue S., Carosella E.D. \& Rouas-Freiss N. (2011) Role of HLA-G in tumor escape through expansion of myeloid-derived suppressor cells and cytokinic balance in favor of Th2 versus Th1/Th17. Blood 117, 7021-7031.

Cao W., Brenner C.A., Alikani M., Cohen J. \& Warner C.M. (1999) Search for a human homologue of the mouse Ped gene. Molecular human reproduction 5, 541-547. Carosella E.D., Rouas-Freiss N., Tronik-Le Roux D., Moreau P. \& LeMaoult J. (2015) HLA-G: An Immune Checkpoint Molecule. Advances in immunology 127, 33-144. Chiang E.Y., Henson M. \& Stroynowski I. (2002) The nonclassical major histocompatibility complex molecule Qa-2 protects tumor cells from NK cell- and lymphokine-activated killer cell-mediated cytolysis. J Immunol 168, 2200-2211. Chiang E.Y. \& Stroynowski I. (2004) A nonclassical MHC class I molecule restricts CTL-mediated rejection of a syngeneic melanoma tumor. J Immunol 173, 4394-4401. Cimino-Mathews A., Foote J.B. \& Emens L.A. (2015) Immune targeting in breast cancer. Oncology (Williston Park) 29, 375-385.

Comiskey M., Domino K.E. \& Warner C.M. (2007) HLA-G is found in lipid rafts and can act as a signaling molecule. Human immunology 68, 1-11.

Comiskey M., Goldstein C.Y., De Fazio S.R., Mammolenti M., Newmark J.A. \& Warner C.M. (2003) Evidence that HLA-G is the functional homolog of mouse Qa-2, the Ped gene product. Human immunology 64, 999-1004.

Curigliano G., Criscitiello C., Gelao L. \& Goldhirsch A. (2013) Molecular pathways: human leukocyte antigen G (HLA-G). Clinical cancer research : an official journal of the American Association for Cancer Research 19, 5564-5571. 
De Fazio S.R. \& Warner C.M. (2007) Activation of T cells by cross-linking Qa-2, the ped gene product, requires Fyn. Am J Reprod Immunol 58, 315-324.

Delort L., Rossary A., Farges M.C., Vasson M.P. \& Caldefie-Chezet F. (2015) Leptin, adipocytes and breast cancer: Focus on inflammation and anti-tumor immunity. Life sciences $140,37-48$.

Djurisic S., Skibsted L. \& Hviid T.V. (2015) A Phenotypic Analysis of Regulatory T Cells and Uterine NK Cells from First Trimester Pregnancies and Associations with HLA-G. Am J Reprod Immunol 74, 427-444.

Dong D.D., Yie S.M., Li K., Li F., Xu Y., Xu G., Song L. \& Yang H. (2012) Importance of HLA-G expression and tumor infiltrating lymphocytes in molecular subtypes of breast cancer. Human immunology 73, 998-1004.

Dushyanthen S., Beavis P.A., Savas P., Teo Z.L., Zhou C., Mansour M., Darcy P.K. \& Loi S. (2015) Relevance of tumor-infiltrating lymphocytes in breast cancer. BMC medicine 13, 202.

Engels C.C., Charehbili A., van de Velde C.J., Bastiaannet E., Sajet A., Putter H., van Vliet E.A., van Vlierberghe R.L., Smit V.T., Bartlett J.M., Seynaeve C., Liefers G.J. \& Kuppen P.J. (2015) The prognostic and predictive value of Tregs and tumor immune subtypes in postmenopausal, hormone receptor-positive breast cancer patients treated with adjuvant endocrine therapy: a Dutch TEAM study analysis. Breast cancer research and treatment 149, 587-596.

Ferreira E., da Silva A.E., Serakides R., Gomes M.G. \& Cassali G.D. (2007) Ehrlich tumor as model to study artificial hyperthyroidism influence on breast cancer. Pathology, research and practice 203, 39-44. 
Jiang F., Zhao H., Wang L., Guo X., Wang X., Yin G., Hu Y., Li Y. \& Yao Y. (2015) Role of HLA-G1 in trophoblast cell proliferation, adhesion and invasion. Biochemical and biophysical research communications 458, 154-160.

Kabel A.M., Omar M.S., Balaha M.F. \& Borg H.M. (2015) Effect of metformin and adriamycin on transplantable tumor model. Tissue \& cell 47, 498-505.

Kreso A. \& Dick J.E. (2014) Evolution of the cancer stem cell model. Cell stem cell $14,275-291$.

Lee M., Choi B., Kwon H.J., Shim J.A., Park K.S., Lee E.S. \& Sohn S. (2010) The role of Qa-2, the functional homolog of HLA-G, in a Behcet's disease-like mouse model induced by the herpes virus simplex. J Inflamm (Lond) 7, 31.

Li C., Houser B.L., Nicotra M.L. \& Strominger J.L. (2009) HLA-G homodimerinduced cytokine secretion through HLA-G receptors on human decidual macrophages and natural killer cells. Proceedings of the National Academy of Sciences of the United States of America 106, 5767-5772.

Li C., Toth I., Schulze Zur Wiesch J., Pereyra F., Rychert J., Rosenberg E.S., van Lunzen J., Lichterfeld M. \& Yu X.G. (2013) Functional characterization of HLA-G(+) regulatory T cells in HIV-1 infection. PLoS pathogens 9, e1003140.

Liu X., Gu W. \& Li X. (2013) HLA-G regulates the invasive properties of JEG-3 choriocarcinoma cells by controlling STAT3 activation. Placenta 34, 1044-1052. Loumagne L., Baudhuin J., Favier B., Montespan F., Carosella E.D. \& Rouas-Freiss N. (2014) In vivo evidence that secretion of HLA-G by immunogenic tumor cells allows their evasion from immunosurveillance. International journal of cancer 135, 2107-2117. Mao Y., Qu Q., Zhang Y., Liu J., Chen X. \& Shen K. (2014) The value of tumor infiltrating lymphocytes (TILs) for predicting response to neoadjuvant chemotherapy in breast cancer: a systematic review and meta-analysis. PloS one 9, e115103. 
Marshall E.A., Ng K.W., Kung S.H., Conway E.M., Martinez V.D., Halvorsen E.C., Rowbotham D.A., Vucic E.A., Plumb A.W., Becker-Santos D.D., Enfield K.S., Kennett J.Y., Bennewith K.L., Lockwood W.W., Lam S., English J.C., Abraham N. \& Lam W.L. (2016) Emerging roles of T helper 17 and regulatory T cells in lung cancer progression and metastasis. Molecular cancer 15, 67.

Matkowski R., Gisterek I., Halon A., Lacko A., Szewczyk K., Staszek U., Pudelko M., Szynglarewicz B., Szelachowska J., Zolnierek A. \& Kornafel J. (2009) The prognostic role of tumor-infiltrating CD4 and CD8 T lymphocytes in breast cancer. Anticancer research 29, 2445-2451.

Mittal D., Gubin M.M., Schreiber R.D. \& Smyth M.J. (2014) New insights into cancer immunoediting and its three component phases--elimination, equilibrium and escape. Current opinion in immunology 27, 16-25.

Morandi F., Rouas-Freiss N. \& Pistoia V. (2014) The emerging role of soluble HLA-G in the control of chemotaxis. Cytokine \& growth factor reviews 25, 327-335.

Naji A., Durrbach A., Carosella E.D. \& Rouas-Freiss N. (2007) Soluble HLA-G and HLA-G1 expressing antigen-presenting cells inhibit T-cell alloproliferation through ILT-2/ILT-4/FasL-mediated pathways. Human immunology 68, 233-239.

Naji A., Le Rond S., Durrbach A., Krawice-Radanne I., Creput C., Daouya M., Caumartin J., LeMaoult J., Carosella E.D. \& Rouas-Freiss N. (2007) CD3+CD4low and CD3+CD8low are induced by HLA-G: novel human peripheral blood suppressor Tcell subsets involved in transplant acceptance. Blood 110, 3936-3948.

Pankratz S., Bittner S., Herrmann A.M., Schuhmann M.K., Ruck T., Meuth S.G. \& Wiendl H. (2014) Human CD4+ HLA-G+ regulatory T cells are potent suppressors of graft-versus-host disease in vivo. FASEB journal : official publication of the Federation of American Societies for Experimental Biology 28, 3435-3445. 
Park G.M., Lee S., Park B., Kim E., Shin J., Cho K. \& Ahn K. (2004) Soluble HLA-G generated by proteolytic shedding inhibits NK-mediated cell lysis. Biochemical and biophysical research communications 313, 606-611.

Richmond A. \& Su Y. (2008) Mouse xenograft models vs GEM models for human cancer therapeutics. Disease models \& mechanisms 1, 78-82.

Rizzo R., Trentini A., Bortolotti D., Manfrinato M.C., Rotola A., Castellazzi M., Melchiorri L., Di Luca D., Dallocchio F., Fainardi E. \& Bellini T. (2013) Matrix metalloproteinase-2 (MMP-2) generates soluble HLA-G1 by cell surface proteolytic shedding. Molecular and cellular biochemistry 381, 243-255.

Romo-Gonzalez T., Esquivel-Velazquez M., Ostoa-Saloma P., Lara C., Zentella A., Leon-Diaz R., Lamoyi E. \& Larralde C. (2015) The network of antigen-antibody reactions in adult women with breast cancer or benign breast pathology or without breast pathology. PloS one 10, e0119014.

Sato E., Olson S.H., Ahn J., Bundy B., Nishikawa H., Qian F., Jungbluth A.A., Frosina D., Gnjatic S., Ambrosone C., Kepner J., Odunsi T., Ritter G., Lele S., Chen Y.T., Ohtani H., Old L.J. \& Odunsi K. (2005) Intraepithelial CD8+ tumor-infiltrating lymphocytes and a high CD8+/regulatory $\mathrm{T}$ cell ratio are associated with favorable prognosis in ovarian cancer. Proceedings of the National Academy of Sciences of the United States of America 102, 18538-18543.

Smalley M., Piggott L. \& Clarkson R. (2013) Breast cancer stem cells: obstacles to therapy. Cancer letters 338, 57-62.

Tung N.M. \& Winer E.P. (2015) Tumor-infiltrating lymphocytes and response to platinum in triple-negative breast cancer. Journal of clinical oncology : official journal of the American Society of Clinical Oncology 33, 969-971. 
Wesolowski R. \& Carson W.E., 3rd (2014) Tumor Infiltrating Lymphocytes - The Next Step in Assessing Outcome and Response to Treatment in Patients with Breast Cancer. Journal of carcinogenesis \& mutagenesis 5.

Yang S.H., Li C.F., Chu P.Y., Ko H.H., Chen L.T., Chen W.W., Han C.H., Lung J.H. \& Shih N.Y. (2016) Overexpression of regulator of G protein signaling 11 promotes cell migration and associates with advanced stages and aggressiveness of lung adenocarcinoma. Oncotarget 7, 31122-31136. 